Research Paper

\title{
Effects of the Geometrical Structure of a Honeycomb TCP on Relationship between Bone / Cartilage Formation and Angiogenesis
}

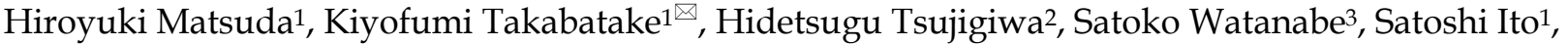 \\ Hotaka Kawai ${ }^{1}$, Mei Hamada ${ }^{1}$, Saori Yoshida ${ }^{1}, K$ Keisuke Nakano ${ }^{1}$, Hitoshi Nagatsuka ${ }^{1 凶}$
}

1. Department of Oral Pathology and Medicine, Graduate School of Medicine, Dentistry and Pharmaceutical Science, Okayama University, Okayama, Japan

2. Department of life science, Faculty of Science, Okayama University of Science, Okayama, Japan

3. Department of Plastic and Reconstructive Surgery, Graduate School of Medicine, Dentistry and Pharmaceutical Science, Okayama University, Okayama, Japan

$\square$ Corresponding author: Hitoshi Nagatsuka, Department of Oral Pathology and Medicine, Graduate School of Medicine, Dentistry and Pharmaceutical Sciences, Okayama University. 2-5-1 Shikata-Cho, Okayama 700-8558, Japan. Phone: (+81) 86-2351-6651, Fax: (+81) 86-235-6654 E-mail: jin@md.okayama-u.ac.jp Kiyofumi Takabatake, Department of Oral Pathology and Medicine, Graduate School of Medicine, Dentistry and Pharmaceutical Sciences, Okayama University. 2-5-1 Shikata-Cho, Okayama 700-8558, Japan. Phone: (+81) 86-2351-6651, Fax: (+81) 86-235-6654 E-mail: gmd422094@s.okayama-u.ac.jp

(c) Ivyspring International Publisher. This is an open access article distributed under the terms of the Creative Commons Attribution (CC BY-NC) license (https://creativecommons.org/licenses/by-nc/4.0/). See http://ivyspring.com/terms for full terms and conditions.

Received: 2018.07.11; Accepted: 2018.09.13; Published: 2018.10.20

\begin{abstract}
A number of biomaterials have been developed, some of which already enjoy widespread clinic use. We have devised a new honeycomb tricalcium phosphate (TCP) containing through-and-through holes of various diameters to control cartilage and bone formation. However, the way in which the geometric structure of the honeycomb TCP controls cartilage and bone tissue formation separately remains unknown. In addition, an association has been reported between bone formation and angiogenesis. Therefore, in the present study, we investigated the relationship between angiogenesis and various hole diameters in our honeycomb TCP over time in a rat ectopic hard tissue formation model. Honeycomb TCPs with hole diameters of 75,300 , and $500 \mu \mathrm{m}$ were implanted into rat femoral muscle. Next, ectopic hard tissue formation in the holes of the honeycomb TCP was assessed histologically at postoperative weeks 1,2, and 3, and CD34 immunostaining was performed to evaluate angiogenesis. The results showed that cartilage formation accompanied by thin and poor blood vessel formation, bone marrow-like tissue with a branching network of vessels, and vigorous bone formation with thick linear blood vessels occurred in the TCPs with $75-\mu \mathrm{m}, 300-\mu \mathrm{m}$, and $500-\mu \mathrm{m}$ hole diameters, respectively. These results indicated that the geometrical structure of the honeycomb TCP affected cartilage and bone tissue formation separately owing to the induced angiogenesis and altered oxygen partial pressure within the holes.
\end{abstract}

Key words: Angiogenesis, Bone formation, Cartilage formation, Geometrical structure, Honeycomb TCP

\section{Introduction}

In recent years, the progression of regenerative medicine has led to the development of various materials. During this process, stem cells, growth factors, and the extracellular matrix (ECM) constitute the elements needed for cell growth and differentiation ${ }^{1-5}$. In addition, it has recently come to be believed that trophic resources (e.g., vessels) and dynamic elements (e.g., mechanical stress) play an important role in cell growth and differentiation ${ }^{6,7}$.
Mesenchymal or induced pluripotent stem cells are often used as the stem cell element, and bone morphogenetic protein-2 (BMP-2), among others, is widely used as a growth factor. Furthermore, in research focusing on the ECM, artificial biomaterials composed of various materials have been researched and developed to reproduce the extracellular microenvironment and induce tissue formation and cell growth and differentiation ${ }^{8-12}$. 
Recently, several studies have focused on the geometrical structure of biomaterials because not only the composition, but also the optimum geometrical structure of artificial biomaterials, is considered important for inducing cell differentiation and tissue formation ${ }^{7,13}$. Regarding hard tissue regeneration in the clinical setting, ceramic biomaterials with high biocompatibility, such as hydroxyapatite and tricalcium phosphate (TCP), have been developed and already enjoy widespread use. Therefore, many researchers have attempted to identify the optimal geometrical structure of artificial biomaterials for inducing hard tissue formation $7,13-15$.

Focusing on the importance of the geometrical structure of artificial biomaterials for inducing cell differentiation and hard tissue formation, we have already succeeded in developing a new honeycomb TCP structure containing through-holes of various diameters. In our previous study, we reported that the difference in surface properties resulting from the sintering temperature affects the biocompatibility and osteoinductivity of $\mathrm{TCP}^{16}$. Furthermore, changing the geometrical structure of honeycomb TCP holes has successfully controlled cartilage and bone formation ${ }^{17}$. In that study, we investigated histologically how differences in the hole diameters $(75,300,500$, and $1600 \mu \mathrm{m})$ of a honeycomb TCP structure with various final contained amount. of BMP-2 $(0,125,250,500$, and $1000 \mathrm{ng}$ ) influenced bone tissue regeneration. Cartilage formation was observed in the honeycomb TCP with a $75-\mu \mathrm{m}$ pore size and a low contained amount of BMP-2 (125 ng) at 3 weeks after implantation into rat femoral muscle. In addition, a bone marrow-like structure was found in the honeycomb TCP with a $300-\mu \mathrm{m}$ pore size and a high contained amount of BMP-2 (1000 ng), and vigorous bone formation was observed in the honeycomb TCP with a $500-\mu \mathrm{m}$ pore size at 3 weeks after implantation into rat femoral muscle. On the other hand, no bone formation was observed in the honeycomb TCP with a $1600-\mu \mathrm{m}$ pore size regardless of BMP-2 concentration and TCP without BMP-2 did not show hard tissue formation at any pore size. These findings suggest that cartilage and bone formation can be controlled by altering the geometric structure of artificial biomaterials; however, the details underlying this mechanism remain unclear.

In recent years, angiogenesis has been found to be important for appropriate bone formation as it supplies cells, oxygen, nutrients, and cytokines to osteoblast progenitor cells; thus, bone formation is thought to occur in conjunction with angiogenesis. ${ }^{18}$.

With this background, in the present study, we analyzed the relationship between hard tissue formation and angiogenesis in TCP holes over time to examine how the geometrical structure of a honeycomb TCP structure affects the differentiation mechanism of bone and cartilage formation. In this experiment, we used the honeycomb TCP with a $75-\mu \mathrm{m}$ pore size and a low contained amount of BMP-2 (125 ng), which specifically recognizes cartilage tissue formation, and the honeycomb TCP with a $300-\mu \mathrm{m}$ or $500-\mu \mathrm{m}$ pore size and a high contained amount of BMP-2 (1000 ng), which specifically identified bone tissue formation.

\section{Materials and Methods}

\section{Animals and implantation procedure}

A total of 14 4-week-old healthy male Wister rats were used in this experiment. All experiments were performed in accordance with Okayama University's Policy on the Care and Use of the Laboratory Animals and approved by the Animal Care and Use Committee. All surgical procedures were performed under general anesthesia in a pain-free state.

\section{Preparation of honeycomb TCP containing BMP-2}

Honeycomb TCP was pressed in a cylindrical mold with a depth of $5 \mathrm{~mm}$ containing through-and-through holes with diameters of $75 \mu \mathrm{m}$ (75TCP), $300 \mu \mathrm{m}$ (300TCP), and $500 \mu \mathrm{m}$ (500TCP). Each TCP was calcinated by heating to $1200{ }^{\circ} \mathrm{C}$ (Fig. 1). Details of TCP manufacturing method have been described previously ${ }^{16}$.

Each TCP structure was sterilized by autoclave and loaded with BMP-2. 75TCP was loaded with BMP-2 diluted to a final contained amount of $125 \mathrm{ng}$ in Matrigel $^{\circledR}$ (BD Biosciences, Inc., NJ, USA), and 300TCP and 500TCP were loaded with BMP-2 diluted to a final contained amount of $1000 \mathrm{ng}$ in Matrigel $^{\circledR}$ (BD Bioscience). Next, these TCPs were implanted into rat femoral muscle.

\section{Histological procedure}

For histological observations, the implanted TCPs were removed after 1, 2, and 3 weeks and fixed in neutral buffered formalin. Next, the specimens were decalcified in 10\% ethylenediaminetetraacetic acid for 2 weeks and then embedded in paraffin. Finally, sections were stained with hematoxylin-eosin (HE) and observed histologically.

\section{CD34 immunostaining}

In the present study, rabbit polyclonal anti-CD34 antibody (Abcam, Tokyo, Japan) was used as a vascular endothelial marker. CD34 is a cell-surface glycoprotein known to have the ability to differentiate into all blood and endothelial cells. For that reason, the presence of CD34 was investigated. 


\section{Hole diameter: $\underline{75 \mu \mathrm{m}}$ Number of holes: 568}
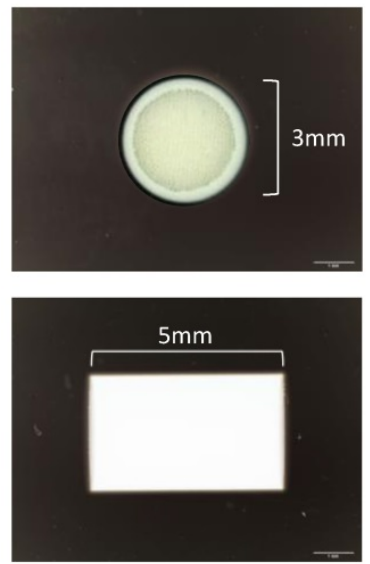

Hole diameter: $300 \mu \mathrm{m}$ Number of holes: $\mathbf{3 7}$
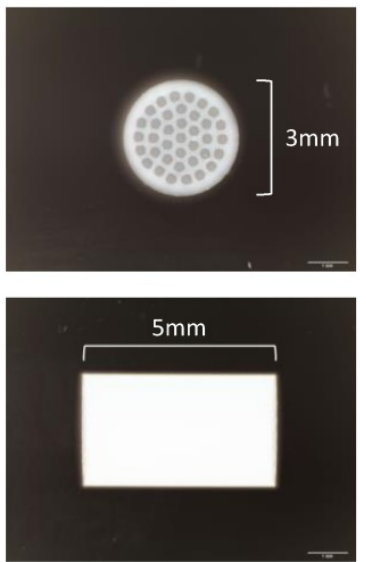

\section{Hole diameter: $\underline{500 \mu m}$ Number of holes: 16}
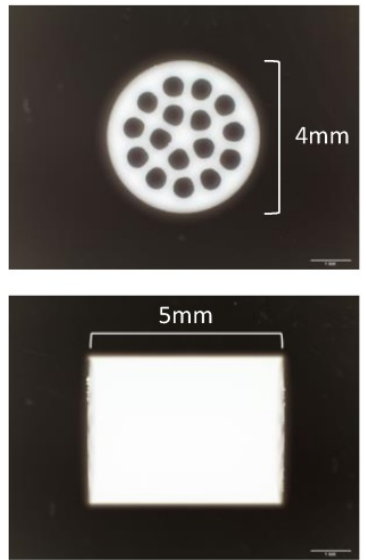

Figure 1. The honeycomb TCP structure used in this experiment.

CD34 immunostaining was carried out as follows. The sections were deparaffinized in a series of xylene solutions for $15 \mathrm{~min}$ and then rehydrated in graded ethanol solutions. Endogenous peroxidase activity was blocked by incubating the sections in $0.3 \% \mathrm{H}_{2} \mathrm{O}_{2}$ in methanol for $30 \mathrm{~min}$. Antigen retrieval was performed in $0.01 \mathrm{~mol} / 1$ citrate buffer $(\mathrm{pH}$ 6.0) for 1 min. After incubation with normal serum, the sections were incubated overnight with primary antibodies at $4{ }^{\circ} \mathrm{C}$. Tagging of primary antibodies was achieved by the subsequent application of anti-rabbit IgG (ABC kit; Vector Laboratories, Inc., Burlingame, CA, USA). Immunoreactivity was visualized using a diaminobenzidine (DAB) $/ \mathrm{H}_{2} \mathrm{O}_{2}$ solution (Histofine DAB substrate; Nichirei, Tokyo, Japan), and slides were counterstained with Mayer's HE (Merck KGaA, Darmstadt, Germany).

\section{Hard tissue and vessel formation evaluation by area measurement}

To quantify the hard tissue area, hard tissue formation was measured in five areas chosen from randomly selected regions in HE-stained specimens (200× magnification, $n=4)$ using Image J software (NIH, Bethesda, MD, USA). In each field, we measured the total area of bone or cartilage formation in TCP holes and the area of TCP holes and we calculated the ratio of area of bone or cartilage area in TCP holes to determine the average of the 5 fields. The obtained average value was compared in each group, the rate of bone formation and cartilage formation were compared for different pore size. To evaluate angiogenesis in the TCP holes of various diameters, vessel number counts per a TCP hole, vessels area measurements in TCP holes in a manner similar to that of the hard tissue, and average vessel thicknesses were evaluated in a TCP hole.

\section{Statistical analysis}

Statistical analysis was performed using one-way analysis of variance and Fisher's exact tests. A $P$ value $<0.05$ was considered statistically significant. All calculations were performed using PASW Statistics 18 (SPSS Inc., Chicago, IL, USA).

\section{Results}

\section{Hard tissue formation in TCP holes over time}

Histological findings in 75TCP over time

At 1 week, cell penetration was seen at the entrance of the 75TCP holes, and small blood vessels were observed penetrating into the pores (Fig. 2a). At 2 weeks, fibrous connective tissue was observed to be filling the holes to the center; however, no hard tissue formation was seen (Fig. 2b). At 3 weeks, chondrogenesis which was positive of Toluidine blue staining was observed to be filling the TCP holes, and angiogenesis was poor compared with that seen at 2 weeks (Fig. 2c).

\section{Histological findings in 300TCP over time}

At 1 week, in the 300TCP holes, invasion and proliferation of fibrous connective tissue were observed in about one-third of the area from the entrance of the TCP holes. In addition, microvessel invasion was observed around the entrance and a cartilage matrix with clear vesicles had formed on the inner walls. Basic staining showed the cartilage matrix to be homogeneous and nonstructural, and Toluidine blue staining positive image was observed to fit the cartilage-like tissue (Fig. 2d). At 2 weeks, cell infiltration and microvessel invasion were observed 
up to the center of the holes, and the formation of woven bone surrounded by osteoblasts was observed around the inner walls (Fig. 2e). At 3 weeks, formation of bone tissue was found around the inner walls. In addition, new bone formation was observed to be covering the inner walls, and a small amount of cancellous trabecular bone formation was observed in the pore cavities. A large number of polygonal osteoblasts were arranged around the bone matrix, suggesting that 300TCP had the highest osteogenic activity. Moreover, the formation of numerous vascular lumens was observed penetrating the lumen surrounded by bone tissue; vascular lumens were even observed in the central part of the TCP. In addition, bone marrow-like tissue with many blood cells was observed in the vascular lumen-rich part of the TCP (Fig. 2f).

\section{Histological findings in 500TCP over time}

At 1 week, in 500TCP holes, as in the case of
300TCP, infiltration of fibrous connective tissue and cells was observed in about one-third of the area from the entrance of the TCP holes. In the vicinity of the entrance, island-shaped cartilage tissue and cartilage formation attached to the walls of the TCP were observed; woven bone formation was also observed in the part following the cartilage matrix which was Toluidine blue staining positive. Moreover, thick straight blood vessel invasion compared with 300TCP was observed (Fig. 2g). At 2 weeks, the proliferation of fibrous connective tissue and the invasion of thick linear blood vessels were observed around the central part of the TCP, and bone formation was found around the thick blood vessels (Fig. 2h). At 3 weeks, 500TCP showed a similar pattern of bone formation as 300TCP. In addition, numerous newly cancellous bone tissues were found; however, no bone marrow-like tissues were found in the area surrounded by these bone tissues (Fig. 2i).
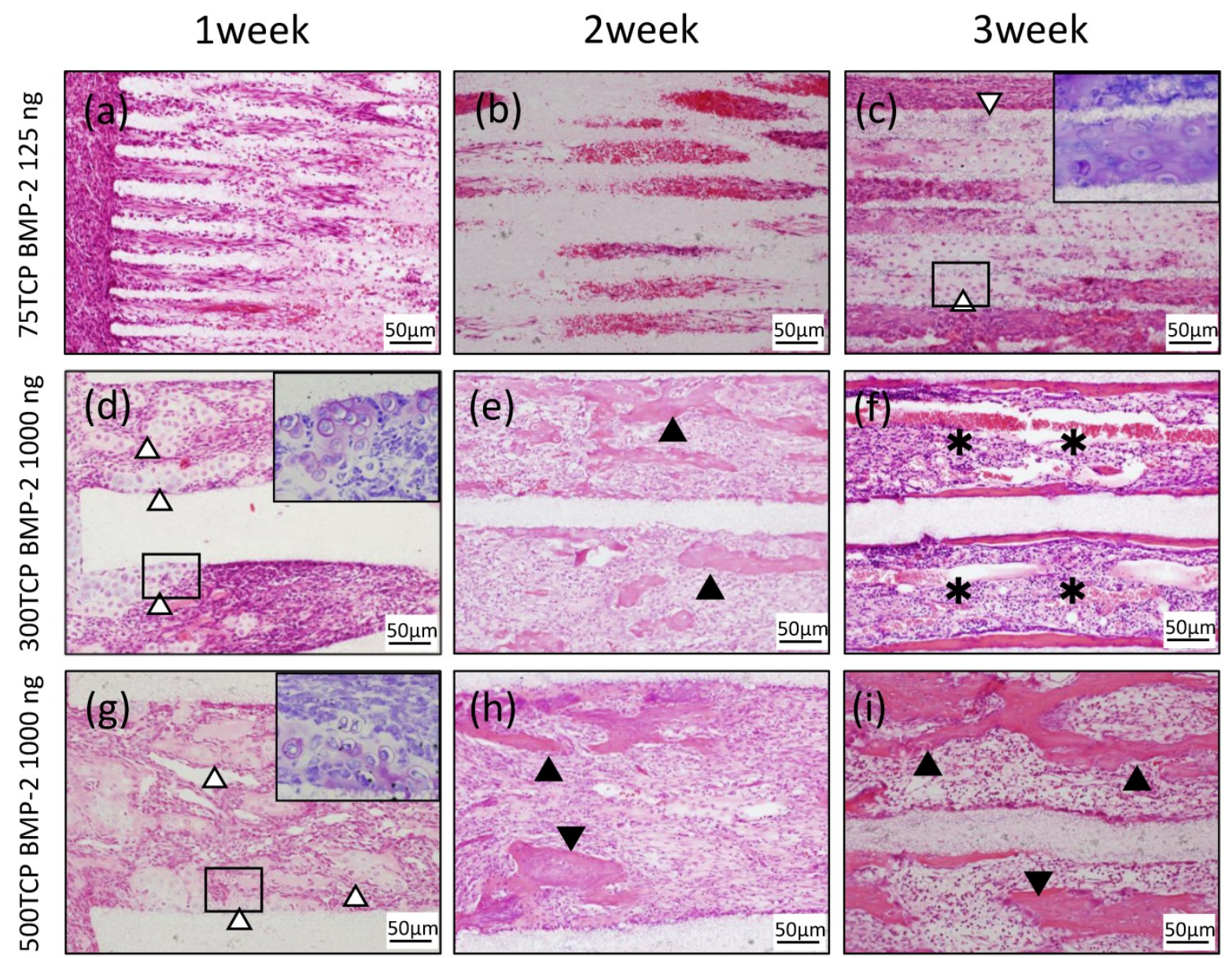

Figure 2. Histological findings. (a) Cell penetration was observed at the entrance of the TCP holes. (b) Fibrous connective tissue was observed filling the TCP holes up to the center, but no hard tissue formation was observed. (c) Chondrogenesis was observed filling the TCP holes (white arrowheads). Inset: Toluidine blue staining positive images. (d) Invasion and proliferation of fibrous connective tissue and cartilage formation (white arrowheads) were observed in about one-third of the area from the entrance of the TCP holes. Inset: Toluidine blue staining positive images. (e) Cell infiltration was observed up to the center of the TCP holes, and bone formation was observed on the inner walls (arrowheads). (f) Formation of bone and bone marrow-like tissue (asterisks) was found in the TCP holes. ( $\mathrm{g}$ ) Infiltration of fibrous connective tissue and cartilage formation (white arrowheads) were observed in about one-third of the area from the entrance of the TCP holes. Inset: Toluidine blue staining positive images. (h) Bone formation (arrowheads) was found around the thick blood vessels. (i) Numerous newly cancellous bone tissues were observed (arrowheads). 


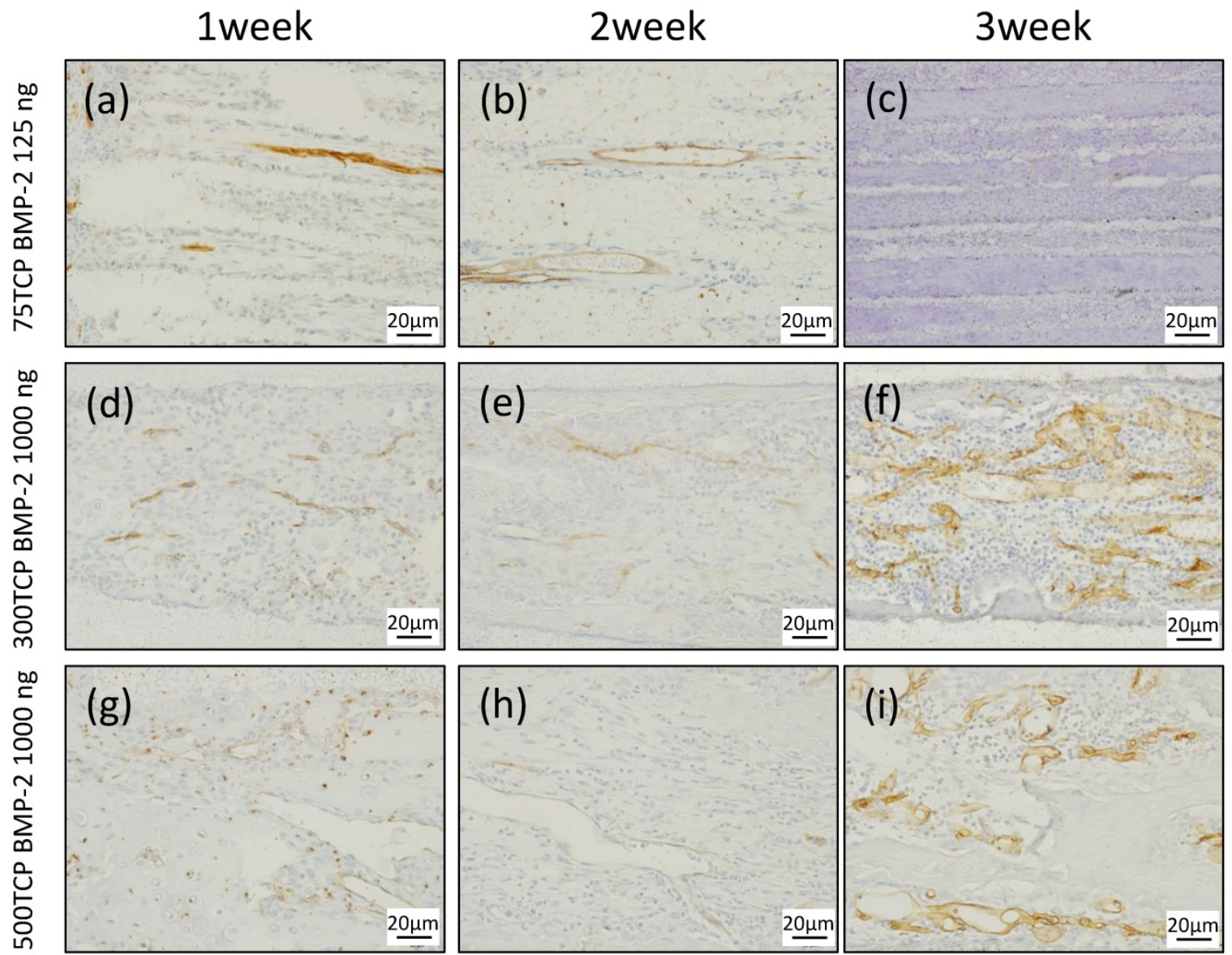

Figure 3. CD34 immunostaining. (a) Small blood vessels were observed in about one-third of the area from the entrance of the TCP holes. (b) Small blood vessels were observed up to the center of the TCP holes. (c) Angiogenesis was poor at 3 weeks compared with that at 2 weeks. (d) Microvessel invasion was also observed around the entrance of the TCP holes. (e) Vascular invasion was observed up to the center of the TCP holes, along with a branching network of blood vessel formation. (f) Blood vessels similar to sinusoidal vessels were observed in the bone marrow-like structures formed within the TCP holes. ( $\mathrm{g}$ ) $\mathrm{A}$ thick formation of vascular invasion was observed in the vicinity of the TCP hole entrances. (h) A plurality of thick rectilinear blood vessels penetrating into the central part of the TCP holes was observed. (i) At 3 weeks, a pattern of blood vessel invasion similar to that at 2 weeks was seen.

\section{Dynamics of blood vessels entering TCP over time}

We investigated the dynamics of blood vessels entering the TCP over time using CD34 immunostaining.

At 1 week, small blood vessel invasion was observed in the vicinity of the 75TCP hole entrances. The amount of vascular invasion was about one per hole (Fig. 3a). At 2 weeks, blood vessel invasion was observed even in the central part of the TCP holes; however, similar to the findings at 1 week, the formation pattern was thin and the amount of vascular invasion was about one per hole (Fig. 3b). At 3 weeks, the insides of the TCP holes were filled with hard tissue composed mainly of cartilage, so the blood vessel invasion was poorer than that seen at 2 weeks (Fig. 3c).

At 1 week, multiple vascular invasions were observed in the vicinity of the 300TCP hole entrances (Fig. 3d). At 2 weeks, vascular invasion was observed around the central part, along with a branching network of blood vessel formation (Fig. 3e). At 3 weeks, blood vessels similar to sinusoidal vessels were observed in the bone marrow-like structures formed within the TCP holes (Fig. 3f).

At 1 week, a thick formation of vascular invasion was observed in the vicinity of the 500TCP hole entrances (Fig. 3g). At 2 weeks, a plurality of thick rectilinear blood vessels penetrating into the central part of the TCP holes was observed (Fig. 3h), and at 3 weeks, a pattern of blood vessel invasion similar to that at 2 weeks was seen (Fig. 3i).

\section{Quantitative examination of hard tissue and vessels. formation in TCP}

To investigate the correlation between hard tissue formation and angiogenesis, we quantitatively examined the area of hard tissue formation and blood vessels invading into the TCP holes over time. Therefore, we analyzed the area, number, and thickness of blood vessels entering into the TCP holes.

The area of bone formation tended to increase in the TCP holes over time, regardless of diameter. The 
amount of bone formation amount tended to increase with increases in hole size from 75 to $500 \mu \mathrm{m}$ (Fig. 4a). By contrast, cartilage formation tended to decrease in 300TCP and 500TCP over time, whereas marked chondrogenesis was observed in 75TCP at 3 weeks (Fig. 4b).

The area of blood vessel invasion tended to increase with increasing hole size over time; however, invasion at 3 weeks decreased compared with that at 2 weeks in 75TCP (Fig. 5a). The number of blood vessels invading into the TCP holes also tended to increase over time and with increasing pore size. In 75TCP, the number of invasive blood vessels was two or fewer per hole, and in each TCP hole, the number of blood vessels was almost about one. In addition, the number of blood vessels decreased at 3 weeks (Fig. 5b). The thickness of the blood vessels entering the TCP holes tended to increase over time and with increasing hole diameters (Fig. 5c).

In 300TCP and 500TCP, the invasive blood vessel and bone tissue areas tended to increase over time, whereas in 75TCP, although hard tissue formation, especially cartilage formation, tended to increase over time, the blood vessel area and thickness decreased at 3 weeks.

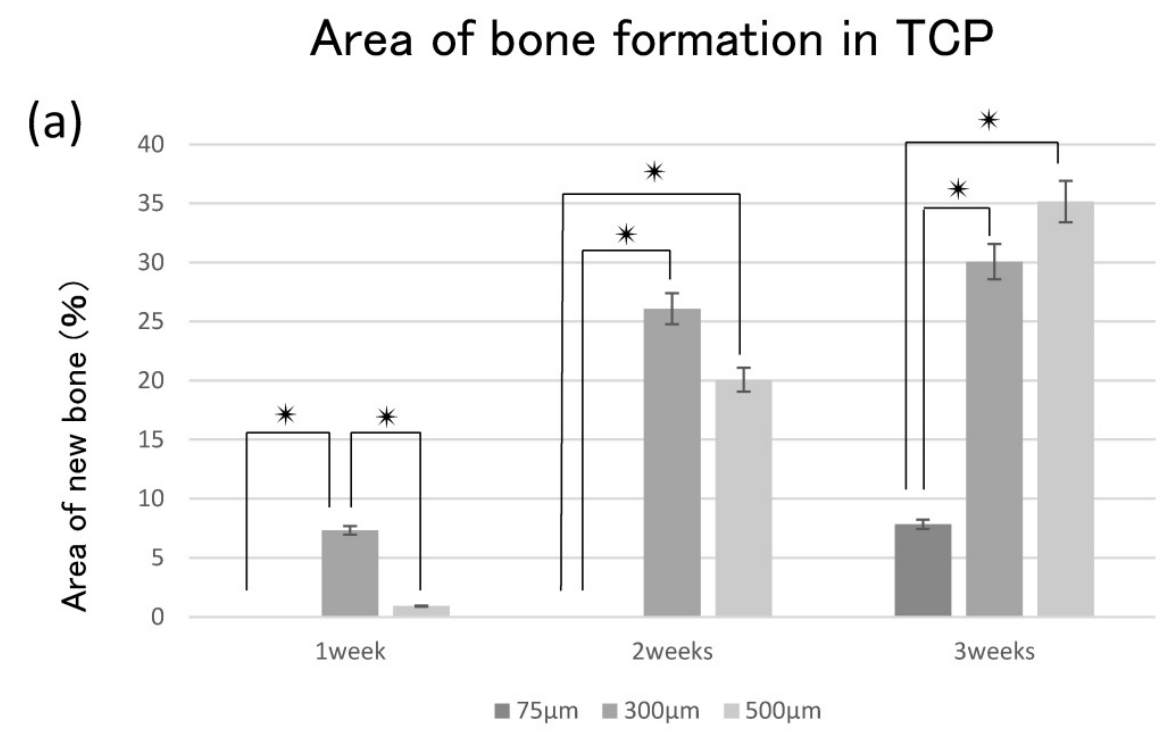

Area of cartilage formation in TCP

(b)

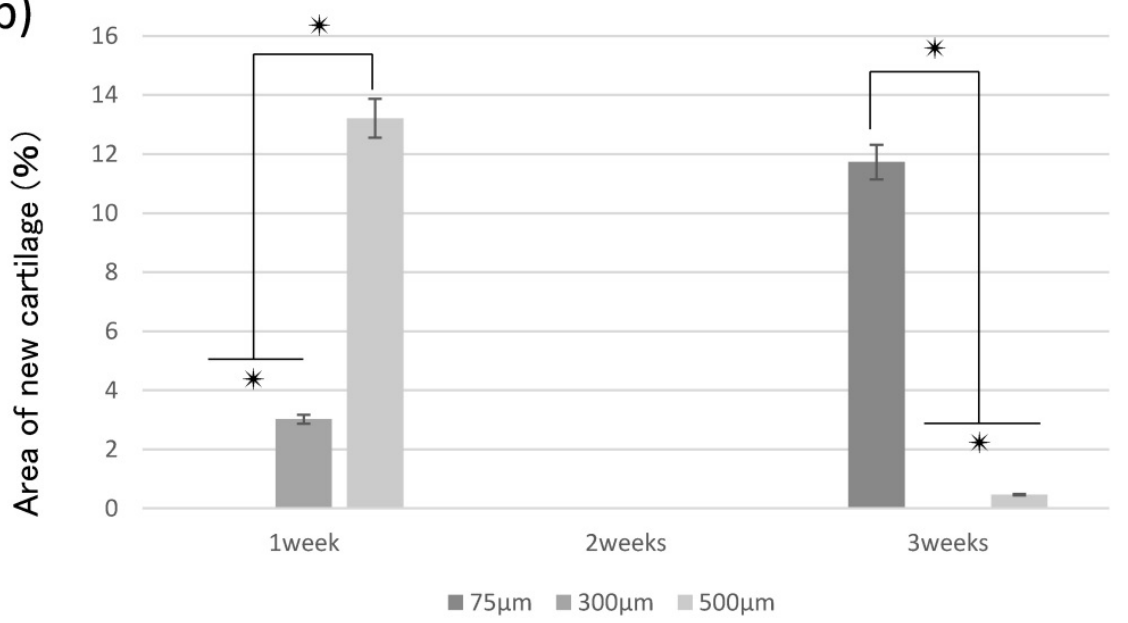

Figure 4. Quantitative analysis of the hard tissue area. (a) Quantification of the neonatal bone tissue area in the TCP holes. (b) Quantification of the neonatal cartilage tissue area in the TCP holes. $\quad *$ : $\mathrm{p}<0.05$ 


\section{Blood vessel formation in TCP}

(a)

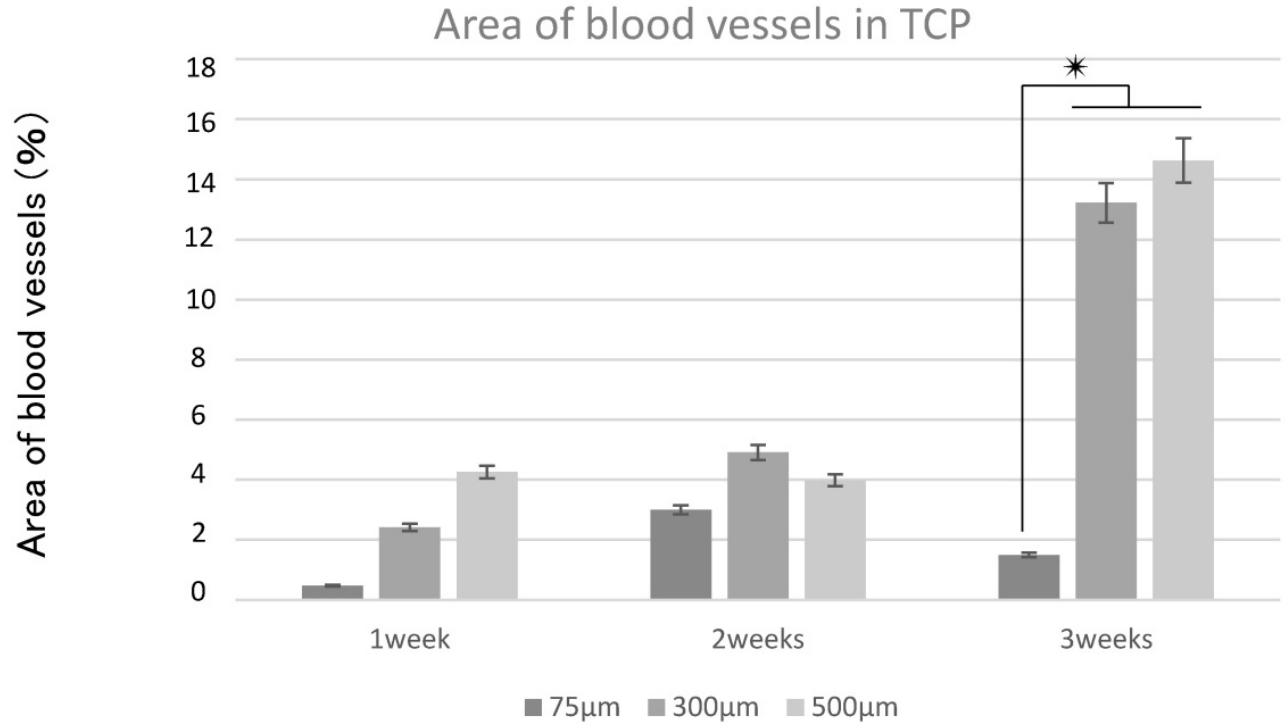

(b)

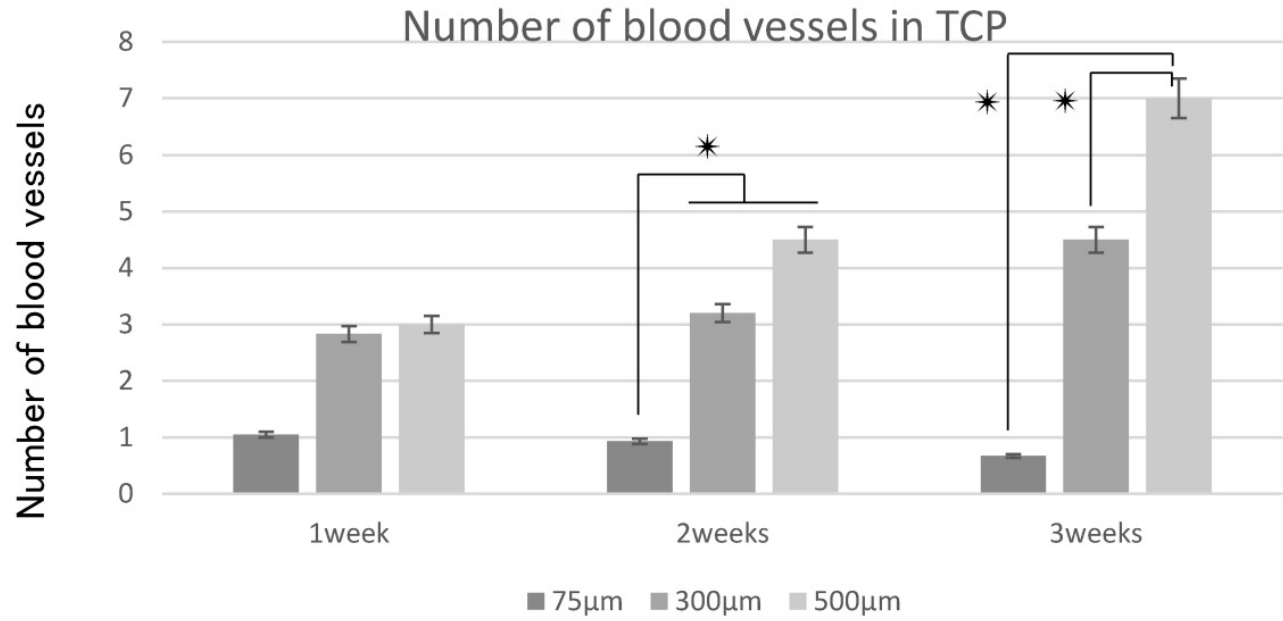

(c)

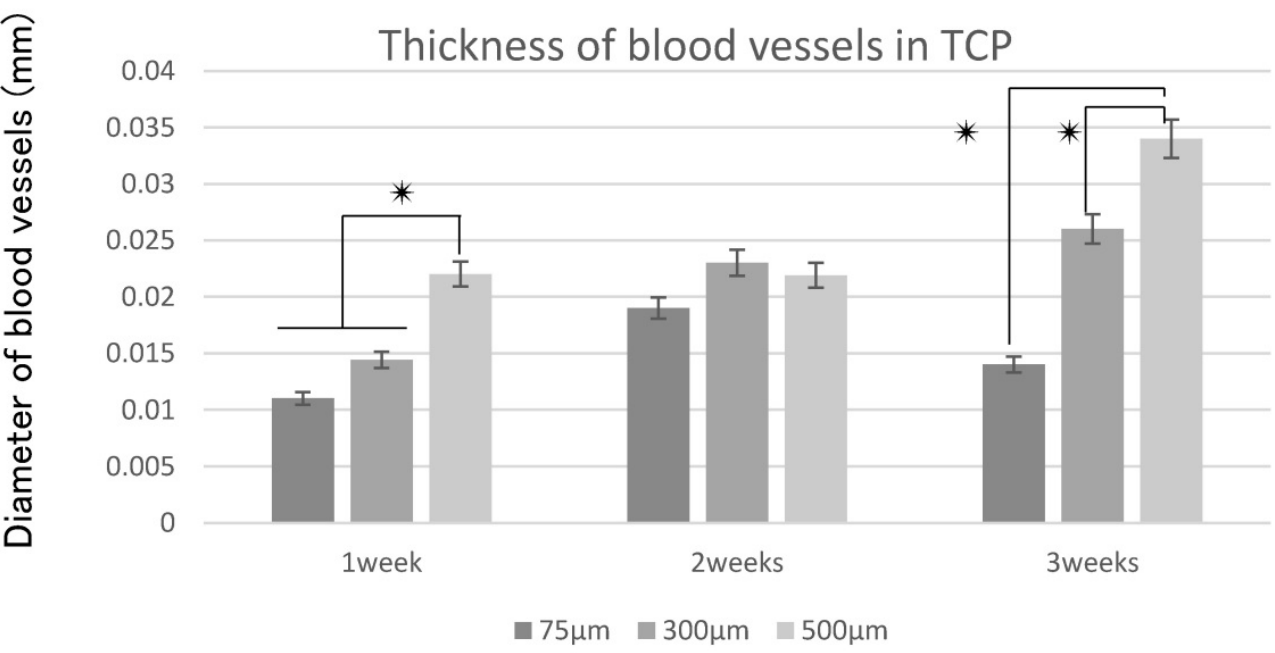

Figure 5. Quantitative analysis of angiogenesis. (a) Quantification of the angiogenesis area in the TCP holes over time. (b) Quantification of the amount of angiogenesis in the TCP holes. (c) Quantification of the diameter of neonatal vessels in the TCP holes. *: $\mathrm{p}<0.05$ 


\section{Discussion}

Stem cells, scaffolds, and growth factors are important for tissue regeneration, and normal tissue regeneration does not occur when any of these elements are missing1-5. Among these elements, artificial biomaterials (scaffolds) provide the environment for cell growth and differentiation. In addition, ideal artificial biomaterials must have an affinity for living tissue, a structure that cells are likely to invade, and tissue solubility ${ }^{19}$.

As a result of CD34 immunostaining, the formation of vascular lumens penetrating the TCP holes was observed in 300TCP and 500TCP. Blood vessel formation is important for not only tissue regeneration, but also bone tissue formation ${ }^{20,21}$. The results of the present experiment also suggested that angiogenesis exerts a substantial influence on bone tissue formation. The invasive blood vessel formation pattern in 300TCP was different from that in 500TCP, which showed the same linear angiogenesis. The reason for this was thought to be the geometric structure of TCP, which influenced the shape of the invasive blood vessels and affected bone formation. Therefore, the results of the present study suggest that the formation of invasive blood vessels was controlled by the pore size of the honeycomb TCP. In addition, the differences in hard tissues formation were thought to be the result of differences in oxygen partial pressure caused by differences in the shape of blood vessels influenced by the geometrical structure of the TCP. Bassett et al. ${ }^{22}$ reported that when mesenchymal stem cells were cultured under various conditions, the precursor cells differentiated into osteoblasts when cultured under high oxygen partial pressure, and into chondrocytes otherwise. The differentiation of the precursor cells was therefore considered to be determined by the environment in which the progenitor cells were placed, particularly in terms of oxygen partial pressure. It has long been known that bone marrow is in a hypoxic environment, and changes in oxygen concentration have been shown to affect the hematopoietic mechanism ${ }^{23,24}$.

In the present study, 500TCP induced vigorous osteogenesis with linear thick blood vessel invasion and 300TCP induced bone marrow formation with fine reticulated vessel invasion, respectively. On the other hand, cartilage formation with narrow blood vessel invasion was observed in 75TCP. Therefore, cartilage, bone marrow, and bone formation occurred in relation to angiogenesis in the TCP holes (in the order of $75 \mathrm{TCP}, 300 \mathrm{TCP}, 500 \mathrm{TCP}$ ), that is, in accordance with decreased oxygen partial pressure. These findings suggest that controlling blood vessel invasion into TCP structures may control the pattern of hard tissue formation. The geometrical structure of the TCP holes with various pore sizes reflected the oxygen partial pressure of the bone, bone marrow, and cartilage tissue environments in the living body, and it appears that these kinds of environments were reproduced by the honeycomb TCP. By changing the pore sizes of the honeycomb TCP, it was possible to reproduce the optimal environment for the desired regeneration of hard tissue.

Our results indicated that angiogenesis decreased at 3 weeks in 75TCP. Chondromodulin produced from chondrocytes contributes to normal cartilage formation by blocking blood vessel invasion ${ }^{25}$. Therefore, chondromodulin secreted from the cartilage tissue filling the holes in 75TCP inhibited angiogenesis and preserved cartilage tissue in 75TCP. In addition, since BMP-2 has been reported to be involved in cell aggregation and angiogenesis 26,27 , if the concentration of BMP-2 is low, so is the capacity for bone formation induced by angiogenesis. Therefore, 75TCP with a low concentration of BMP-2 is advantageous for cartilage formation.

In conclusion, the results of the present study indicated that the linear geometry of our honeycomb TCP structure promoted angiogenesis and hard tissue formation. Therefore, by altering the pore size and controlling blood vessel invasion, our honeycomb TCP structure may allow the selective and efficient formation of cartilage tissue and bone.

\section{Acknowledgments}

This study was funded by the Japan Society for Promotion of Science (JSPS) KAKENHI Grant-in-Aid for Scientific Research (No. 16K20577) and (No. 18K17224).

\section{Conflict of Interest}

The authors declare that they have no conflict of interest.

\section{References}

1. Reddi AH, Huggins CB. Infuence of geometry of transplanted tooth and bone on transformation of broblasts. Proc Soc Exp Biol Med. 1973; 143: 634-637.

2. Karageorgiou V, Kaplan D. Porosity of 3D biomaterial scaffolds and osteogenesis. Biomaterials. 2005; 26(27): 5474-5491.

3. Burg KJ, Porter S, Kellam JF. Biomaterial developments for bone tissue engineering. Biomaterials. 2000; 21(23): 2347-2359.

4. Stevens M. Biomaterials for bone tissue engineering. Materials Today. 2008; 11(5): 18-25.

5. Langer R, Vacanti JP. Tissue engineering. Science. 1993; 260(5110): 920-926.

6. Zhang L, Liu W, Zhao J, Ma X, Shen L, Zhang Y, Jin F, Jin Y. Mechanical stress regulates osteogenic differentiation and RANKL/OPG ratio in periodontal ligament stem cells by the Wnt/ $\beta$-catenin pathway. Biochim Biophys Acta. 2016; 1860(10): 2211-2219.

7. Kuboki Y, Jin Q, Takita H. Geometry of carriers controlling phenotypic in BMP-induced osteogenesis and condrogenesis. J Bone Joint Surg. 2001; 83A: S1-105-114.

8. Kawakubo A, Matsunaga T, Ishizaki H, Yamada S, Hayashi Y. Zinc as an essential trace element in the acceleration of matrix vesicles-mediated mineral deposition. Microsc Res Tech 2011;74:1161-1165. 
9. Porter JR, Henson A, Popat KC. Biodegradable poly(e-caprolactone)nanowires for bone tissue engineering applications. Biomateri-als 2009; 30:780-788.

10. Arpornmaeklong $\mathrm{P}$, Pripatnanont $\mathrm{P}$, Suwatwirote N. Properties of chitosan-collagen sponges and osteogenic differentiation of rat-bone-marrow stromal cells. Int J Oral Maxillofac Surg 2008; 37:357-366.

11. Abarrategi A, Moreno-Vicente C, Ramos V, Aranaz I, Sanz Casado JV, Lopez-Lacomba JL. Improvement of porous b-TCP scaffolds with rhBMP-2 chitosan carrier film for bone tissue application. Tissue Eng A 2008; 14:1305-1319.

12. Wiria FE, Chua CK, Leong KF, Quah ZY, Chandrasekaran M, Lee MW. Improved biocomposite development of poly(vinyl alcohol)-and hydroxyapatite for tissue engineering scaffold fabrication using selective laser sintering. J Mater Sci Mater Med 2008; 19:989-996.

13. Jin QM, Takita H, Kohgo T, Atsumi K, Itoh H, Kuboki Y. Effect of geometry of hydroxyapatite as a cell substratum in BMP-induced ectopic bone formation. J Biomed Mater Res. 2000; 51: 491-499.

14. Tsuruga E. Pore size of porous hydroxyapatite as the cellsubstratum controls BMP-induced osteogenesis. J Biochem. 1997; 121: 317-324

15. Kiba H, Kuboyama N, Uchida R, Ishizaki $T$, Nishiyama N. Bone ingrowth into the parallel cylindrical tubes with different sizes of porous hydroxyapatite implanted into the rabbits. J Hard Tissue Biol. 2012; 21: 307-314.

16. Takabatake K, Yamachika E, Tsujigiwa H, Takeda Y, Kimura M, Takagi S, Nagatsuka H, Iida S. Effect of geometry and microstructure of honeycomb TCP scaffolds on bone regeneration. J Biomed Mater Res A. 2013; 102(9): 2952-2960.

17. Watanabe S, Takabatake $\mathrm{K}$, Tsujigiwa H, Watanabe $\mathrm{T}$, Tokuyama E, Ito $\mathrm{S}$, Nagatsuka H, Kimata Y. Efficacy of Honeycomb TCP-induced Microenvironment on Bone Tissue Regeneration in Craniofacial Area. Int J Med Sci. 2016; 13(6): 466-476.

18. Kusumbe AP, Ramasamy SK, Adams RH. Coupling of angiogenesis and osteogenesis by a specific vessel subtype in bone. Nature. 2014; 507: 323-328.

19. Yang HY, Thompson I, Yang SF, Chi XP, Evans JR, Cook RJ. Dis-solution characteristics of extrusion free formed hydroxyapatite-tricaicium phosphate scaffolds. J Mater Sci Mater Med 2008; 19:3345-3353

20. Petersen W, Tillmann B. Structure and vascularization of the cruciate ligaments of the human knee joint. Anat Embryol. 1999; 200: 325-334.

21. Hossler FE, Douglas JE. Vascular Corrosion Casting: Review of Advantages and Limitations in the Application of Some Simple Quantitative Methods. Microsc Microanal. 2001; 7: 253-264.

22. Bassett C, Herrmann I. Influence of oxygen concentration and mechanical factors on differentiation of connective tissues in vitro. Nature. 1961; 190: 460-461.

23. Guitart AV, Hammoud $M$, Dello Sbarba $P$, Ivanovic $Z$, Praloran V. Slow-cycling/quiescence balance of hematopoietic stem cells is related to physiological gradient of oxygen. Exp Hematol. 2010; 38: 847-851.

24. Harrison JS, Rameshwar P, Chang V, Bandari P. Oxygen saturation in the bone marrow of healthy volunteers. Blood. 2001; 99(1): 394.

25. Hiraki $Y$, Inoue $H$, Iyama $K$, Kamizono A, Ochiai M, Shukunami C, Iijima S, Suzuki F, Kondo J. Identification of Chondromodulin I as a Novel Endothelial Cell Growth Inhibitor. J.Biol. Chem. 1997; 272: 32419-32426.

26. Chen WC, Chung CH, Lu YC, Wu MH, Chou PH, Yen JY, Lai YW, Wang GS, Liu SC, Cheng JK, Wu YJ, Yeh HI, Wang LY, Wang SW. BMP-2 induces angiogenesis by provoking integrin a6 expression in human endothelial progenitor cells. Biochem Pharmacol. 2018; 150: 256-266.

27. Pan Y, Chen I, Yu Y, Dai K, Wang J, Liu C. Biomater Sci. Enhancement of BMP-2-mediated angiogenesis and osteogenesis by 2-N,6-O-sulfated chitosan in bone regeneration. Biomater Sci. 2018; 6(2): 431-439. 